\title{
Good Ideals in Artinian Gorenstein Local Rings Obtained by Idealization
}

\author{
Shiro GOTO and Satoshi HARAIKAWA
}

Meiji University

\section{Introduction.}

The purpose of this note is to prove the following, which gives a structure theorem of certain ideals in Artinian Gorenstein local rings obtained by idealization.

THEOREM 1.1. Let $R$ be an Artinian local ring with the maximal ideal $\mathfrak{n}$ and let $E=$ $E_{R}(R / \mathfrak{n})$ denote the injective envelope of $R / \mathfrak{n}$. Let $A=R \ltimes E$ be the idealization of $E$ over $R$ and let $I$ be an ideal in $A$. Then the following two conditions are equivalent.

(1) $I=(0): I$.

(2) There exists a pair $(\mathfrak{a}, h)$, where $\mathfrak{a}$ is an ideal in $R$ with $\mathfrak{a}^{2}=(0)$ and $h: L:=$ (0) $: E \mathfrak{a} \rightarrow R / \mathfrak{a}$ is a homomorphism of $R / \mathfrak{a}$-modules, satisfying the following four conditions:

(a) $h(x) h(y)=0$ and $h(x) y+h(y) x=0$ for all $x, y \in L$.

(b) Let $a, b \in R$. Then $a b=0$ if $\bar{a}, \bar{b} \in h(L)$. (Here $\bar{*}$ denotes the reduction $\bmod \mathfrak{a}$.)

(c) Let $a \in R$ with $\bar{a} \in h(L)$. Then ax $\in L$ and $h(a x)=0$ for all $x \in E$.

(d) $I=\{(a, x) \mid a \in R, x \in L$ such that $\bar{a}=h(x)\}$.

When this is the case, the pair $(\mathfrak{a}, h)$ is uniquely determined by $I$ and $\mathfrak{a}=f^{-1}(I)$, where $f: R \rightarrow A, f(a)=(a, 0)$ denotes the structure map.

Let $A$ be a Gorenstein local ring with the maximal ideal $\mathfrak{m}$ and let $I$ be an $\mathfrak{m}$-primary ideal in $A$. Then, following [GIW], we say that $I$ is a good ideal in $A$, if $I$ contains a parameter ideal $Q$ in $A$ as a reduction and the associated graded ring $\mathrm{G}(I)=\bigoplus_{n \geq 0} I^{n} / I^{n+1}$ of $I$ is a Gorenstein ring with $\mathrm{a}(\mathrm{G}(I))=1-\operatorname{dim} A$, where $\mathrm{a}(\mathrm{G}(I))$ denotes the a-invariant of $\mathrm{G}(I)$ ([GW, Definition 3.1.4]). This is a condition equivalent to saying that $I^{2}=Q I$ and $I=Q: I$, that is $I^{2}=Q I$ and the equality $\ell_{A}(A / I)=\frac{1}{2} \ell_{A}(A / Q)$ holds true ([GIW, Propositon 2.2]), where $\ell_{A}(*)$ stands for the length. Therefore, the first condition (1) in Theorem 1.1 is just equivalent to saying that $I$ is a good ideal in $A=R \ltimes E$. In [GIW] the first author, Iai, and Watanabe intensively studied general Gorenstein local rings of arbitrary dimension and established many interesting results on good ideals. Nevertheless, in our very special situation

Received May 16, 2002

1991 Mathematics Subject Classification. Primary 13A30, Secondary $13 \mathrm{H} 10$.

Key words and phrases. idealization, associated graded ring, Gorenstein ring.

The first author is supported by the Grant-in-Aid for Scientific Researches in Japan (C(2), No. 13640044). 
where $A=R \ltimes E$ is an Artinian ring obtained by idealization, the structure of good ideals remains mysterious and no effective classification theorem is known. Our theorem $1.1 \mathrm{might}$ have some significance from this viewpoint. Also, it has influenced a further development [GIK] of the theory of good ideals in higher-dimensional Gorenstein local rings obtained by idealization.

The proof of Theorem 1.1 will be given in Section 3. In Section 4 we shall analyze a concrete example. Let $R$ and $E$ be as are in Theorem 1.1 and let $\mathfrak{a}$ be an ideal in $R$ with $\mathfrak{a}^{2}=(0)$. We put $L=(0):_{E} \mathfrak{a}$. Then, letting $h=0$ in condition (2) of Theorem 1.1, we get that $\mathfrak{a} \times L$ is a good ideal in $A$, whence so is the ideal $\alpha(\mathfrak{a} \times L)$ for all $\alpha \in \operatorname{Aut}_{R \text {-alg }} A$, where Aut $_{R \text {-alg }} A$ denotes the group of automorphisms of the $R$-algebra $A$. Therefore, it seems rather natural to expect that the set $\mathcal{X}_{A}$ of good ideals in $A$ consists of those ideals $I$ of the form $I=\alpha(\mathfrak{a} \times L)$. However, this is not the case in general, which we will show in Section 4 . For that purpose we need some basic facts on the structure of the group Aut $_{R \text {-alg }} A$, which we shall briefly summarize in Section 2.

\section{The structure of the group Aut $_{R-a l g} A$.}

In what follows, let $R$ be an Artinian local ring with the maximal ieal $\mathfrak{n}$ and let $E=$ $\mathrm{E}_{R}(R / \mathfrak{n})$ be the injective envelope of $R / \mathfrak{n}$. Let $A=R \ltimes E$ denote the idealization of $E$ over $R$. Hence $A=R \oplus E$ as $R$-modules and the multiplication in $A$ is given by $(a, x)$. $(b, y)=(a b, a y+b x)$. The ring $A$ is actually an Artinian Gorenstein local ring with the maximal ideal $\mathfrak{m}=\mathfrak{n} \times E([\mathrm{R}])$ and $I=(0) \times E$ is an ideal in $A$ with $I^{2}=(0)$. Let $f: R \rightarrow A, f(a)=(a, 0)$ denote the $R$-algebra structure of $A$.

The purpose of this section is to summarize some results on the structure of the group Aut $_{R \text {-alg }} A$ of automorphisms. Let $p_{1}: A \rightarrow R$ and $p_{2}: A \rightarrow E$ be the maps defined by $p_{1}(a, x)=a$ and $p_{2}(a, x)=x$. Let $\alpha: A \rightarrow A$ be an endmorphism of $R$-algebras. We put $\theta=p_{1} \alpha i$ and $\tau=p_{2} \alpha i$, where $i: E \rightarrow A$ denotes the map defined by $i(x)=(0, x)$. Then we have that $\theta \in \operatorname{Hom}_{R}(E, R), \tau \in \operatorname{Hom}_{R}(E, E)$, and $\alpha(a, x)=(a+\theta(x), \tau(x))$ for all $(a, x) \in A$. Because $(0, x)(0, y)=0$, we furthermore have that

(1) $\theta(x) \theta(y)=0$ and

(2) $\theta(x) \tau(y)+\theta(y) \tau(x)=0$

for all $x, y \in E$. Let $\mathcal{M}$ be the set of pairs $(\theta, \tau)$ where $\theta \in \operatorname{Hom}_{R}(E, R)$ and $\tau \in$ $\operatorname{Hom}_{R}(E, E)$ satisfying the above conditions (1) and (2). Then $\mathcal{M}$ forms a monoid under the operation $(\theta, \tau) \cdot\left(\theta_{1}, \tau_{1}\right)=\left(\theta_{1}+\theta \tau_{1}, \tau \tau_{1}\right)$ with $e=\left(0,1_{E}\right)$ the identity. Let $\mathrm{GL}_{R} E$ denote the group of automorphisms of the $R$-module $E$. Then $(\theta, \tau) \in \mathcal{M}$ is invertible if and only if $\tau \in \mathrm{GL}_{R} E$. When this is the case, we have that $(\theta, \tau)^{-1}=\left(-\theta \tau^{-1}, \tau^{-1}\right)$. Let $\mathcal{G}$ denote the group of invertible elements in $\mathcal{M}$ and let $\Phi: \operatorname{End}_{R \text {-alg }} A \rightarrow \mathcal{M}$ be the map defined by $\Phi(\alpha)=\left(p_{1} \alpha i, p_{2} \alpha i\right)$. Then $\Phi$ is an isomorphism of monoids and gives rise to an isomorphism

$$
\varphi: \operatorname{Aut}_{R-\text { alg }} A \stackrel{\sim}{\rightarrow} \mathcal{G}
$$


of groups as well. Let $\mathcal{H}$ denote the $R$-submodule of $\operatorname{Hom}_{R}(E, R)$ consisting of the elements $\theta$ satisfying the conditions $\theta(x) \theta(y)=0$ and $\theta(x) y+\theta(y) x=0$ for all $x, y \in E$. We put $\mathcal{N}=\left\{\left(\theta, 1_{E}\right\} \mid \theta \in \mathcal{H}\right\}$ and $\mathcal{K}=\left\{(0, \tau) \mid \tau \in \mathrm{GL}_{R} E\right\}$. Then $\mathcal{H} \cong \mathcal{N}$ and $\mathcal{N} \triangleleft \mathcal{G}$, since $\left(\theta_{1}, \tau_{1}\right)^{-1}\left(\theta, 1_{E}\right)\left(\theta_{1}, \tau_{1}\right)=\left(\theta \tau_{1}, 1_{E}\right)$. Therefore, the group $\mathcal{G}$ is a semidirect product of the subgroups $\mathcal{N}$ and $\mathcal{K}$, because $\mathcal{N} \cap \mathcal{K}=\{e\}$ and $(\theta, \tau)=\left(\theta, \tau^{-1}, 1_{E}\right)(0, \tau)$. Let $R^{\times}$denote the group of units in $R$. Then $R^{\times} \cong \mathcal{K}$ since $R^{\times} \cong \mathrm{GL}_{R} E$ (cf. [BH, Proposition 3.2.12 (e)]), so that we have by (2.1) the following.

Proposition 2.2. The group $\mathrm{Aut}_{R-\text { alg }} A$ is a semidirect product of $\mathcal{H}$ and $R^{\times}$.

\section{Proof of Theorem 1.1.}

Let $\mathcal{X}_{A}$ denote the set of good ideals in $A=R \ltimes E$. The purpose of this section is to prove Theorem 1.1. We begin with the following lemma 3.1. Notice that $\ell_{A}(M)=\ell_{R}(M)$ for any $A$-module $M$, since $R / \mathfrak{n}=A / \mathfrak{m}$. We have $\ell_{A}(A)=2 \ell_{R}(R)$, because $\ell_{R}(E)=\ell_{R}(R)$ (cf. [BH, Proposition 3.2.12 (b)]). Hence an ideal $I$ in $A$ is good if and only if $I^{2}=(0)$ and $\ell_{R}(I)=\ell_{R}(R)$ (cf. [GIW, Proposition 2.2]).

LEMMA 3.1. Let $\mathfrak{a}$ be an ideal in $R$ with $\mathfrak{a}^{2}=(0)$ and put $L=(0):_{E}$ a. Then $a \times L \in \mathcal{X}_{A}$.

Proof. It is routine to check that $\mathfrak{a} \times L$ is an ideal in $A$ with $(\mathfrak{a} \times L)^{2}=(0)$. Because $\ell_{R}(L)=\ell_{R}\left(\operatorname{Hom}_{R}(R / \mathfrak{a}, E)\right)=\ell_{R}(R / \mathfrak{a})$ ([BH, Proposition 3.2.12(b)]), we have that $\ell_{R}(\mathfrak{a} \times L)=\ell_{R}(\mathfrak{a})+\ell_{R}(L)=\ell_{R}(\mathfrak{a})+\ell_{R}(R / \mathfrak{a})=\ell_{R}(R)$, whence $\mathfrak{a} \times L \in \mathcal{X}_{A}$.

For each $\theta \in \operatorname{Hom}_{R}(E, R)$ we put $I_{\theta}=\{(\theta(x), x) \mid x \in E\}$. Then $I_{\theta}$ is an $R$-submodule of $A$. We have that $\theta=\theta^{\prime}$, once $I_{\theta} \subseteq I_{\theta^{\prime}}$.

LEMMA 3.2. Let $\theta \in \operatorname{Hom}_{R}(E, R)$. Then the following conditions are equivalent.

(1) $\theta \in \mathcal{H}$.

(2) $v w=0$ for all $v, w \in I_{\theta}$.

(3) $I_{\theta} \in \mathcal{X}_{A}$.

(4) $I_{\theta}=\alpha((0) \times E)$ for some $\alpha \in$ Aut $_{R-a l g} A$.

Proof. (1) $\Rightarrow$ (4) By (2.1) we may choose $\alpha \in \operatorname{Aut}_{R-\text { alg }} A$ so that $\varphi(\alpha)=\left(\theta, 1_{E}\right)$. Then $\alpha((0) \times E)=\{(\theta(x), x) \mid x \in E\}=I_{\theta}$.

(4) $\Rightarrow$ (3) This is clear, since (0) $\times E \in \mathcal{X}_{A}$ by (3.1).

(3) $\Rightarrow(2) \quad$ This is clear, since $I_{\theta}^{2}=(0)$.

(2) $\Rightarrow$ (1) Let $x, y \in E$. Then because $(\theta(x), x)(\theta(y), y)=0$ by assumption (2), we get that $\theta(x) \theta(y)=0$ and $\theta(x) y+\theta(y) x=0$, whence $\theta \in \mathcal{H}$.

COROllary 3.3. The set $\mathcal{X}_{A}$ is infinite, if so is $\mathcal{H}$.

COROLLARY 3.4. Suppose that $R$ is a Gorenstein ring containing an infinite field $k$ of ch $k=2$. Then $\mathcal{X}_{A}$ is infinite, if $R$ is not a field. 
Proof. We take $E=R$ and put $\mathcal{Z}=\left\{z \in R \mid z^{2}=0\right\}$. Then $\mathcal{H} \cong \mathcal{Z}$ as $R$-modules. Therefore the set $\mathcal{H}$ is infinite, because $\mathcal{Z} \neq(0)$ and the field $k$ is infinite. Hence $\mathcal{X}_{A}$ is infinite by (3.3).

Proposition 3.5. Let I be an ideal in A. Then the following conditions are equivalent.

(1) $I \in \mathcal{X}_{A}$ and $f^{-1}(I)=(0)$.

(2) $I=I_{\theta}$ for some $\theta \in \mathcal{H}$.

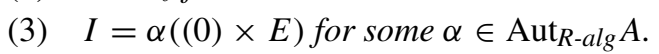

PROOF. $\quad(1) \Rightarrow(2) \quad$ Since $f^{-1}(I)=(0)$, we get the monomorphism $g: I \stackrel{j}{\rightarrow} A \stackrel{p_{2}}{\longrightarrow}$ $E$ (here $j$ denotes the embedding). Since $\ell_{R}(I)=\ell_{R}(E)$, the map $g$ is an isomorphism and so, letting $\theta: E \stackrel{g^{-1}}{\longrightarrow} I \stackrel{j}{\rightarrow} A \stackrel{p_{1}}{\rightarrow} R$, we have $I=I_{\theta}$. Hence $\theta \in \mathcal{H}$ by (3.2).

(2) $\Rightarrow$ (3) See (3.2)

(3) $\Rightarrow(1) \quad$ Since $(0) \times E \in \mathcal{X}_{A}$ by (3.1), we have $I \in \mathcal{X}_{A}$, while $f^{-1}(I)=(0)$ because $f^{-1}((0) \times E)=(0)$.

We are now in a position to prove Theorem 1.1.

Proof OF THEOREM 1.1. (1) $\Rightarrow$ (2) Let $\mathfrak{a}=f^{-1}(I)$ and put $L=(0):_{E} \mathfrak{a}$. Then $\mathfrak{a}^{2}=(0)$ because $I^{2}=(0)$. We have that $x \in L$ if $(a, x) \in I$, since $\mathfrak{a} I=(0)$. Let $\bar{R}=R / \mathfrak{a}$ and $B=\bar{R} \ltimes L$. Then $B$ is a Gorenstein ring, since $L=E_{\bar{R}}(R / \mathfrak{n})$. We denote by $\sigma: I \rightarrow B$ the homomorphism of $R$-modules defined by $\sigma(a, x)=(\bar{a}, x)$ and put $J=\sigma(I)$. Then $J$ is an ideal in $B$ with $J^{2}=(0)$. We have $\ell_{R}(J)=\ell_{R}(I)-\ell_{R}(\mathfrak{a})=\ell_{R}(R / \mathfrak{a})$ because $\operatorname{Ker} \sigma=\mathfrak{a}$ and $\ell_{R}(I)=\ell_{R}(R)$, whence $J \in \mathcal{X}_{B}$. Consequently, because $\bar{f}^{-1}(J)=(0)$ where $\bar{f}: \bar{R} \rightarrow B$ denotes the map defined by $\bar{f}(\bar{a})=(\bar{a}, 0)$, by (3.5) we may write $J=\{(h(x), x) \mid x \in L\}$ with a homomorphism $h: L \rightarrow \bar{R}$ of $\bar{R}$-modules that satisfies the conditions $h(x) h(y)=0$ and $h(x) y+h(y) x=0$ for all $x, y \in L$. Let $(a, x) \in A$ with $(\bar{a}, x) \in J$ and choose $(b, x) \in I$ so that $\bar{a}=\bar{b}$. Then $(a, x)=(b, x)+(a-b, 0) \in I$ because $a-b \in \mathfrak{a}$. Consequently $I=\{(a, x) \mid a \in R, x \in L$ such that $\bar{a}=h(x)\}$. Let $a, b \in R$ and $x, y \in L$ and assume that $\bar{a}=h(x)$ and $\bar{b}=h(y)$. Let $z \in E$. Then because $(a, x),(b, y) \in I$, we have $(a, x)(b, y)=(a b, a y+b x)=0$ and $(0, z)(a, x)=(0, a z) \in I$. Hence $a b=0$ and $a z \in L$ with $h(a z)=0$.

(2) $\Rightarrow(1) \quad$ It is routine to check that $I$ is an ideal in $A$ with $I^{2}=(0)$. Thanks to the exact sequence $0 \rightarrow \mathfrak{a} \stackrel{f^{\prime}}{\rightarrow} I \stackrel{g}{\rightarrow} L \rightarrow$ of $R$-modules (here $f^{\prime}$ and $g$ respectively denote the homomorphisms induced from $f$ and $p_{2}$ ), we have that $\ell_{R}(I)=\ell_{R}(\mathfrak{a})+\ell_{R}(L)=\ell_{R}(R)$. Hence $I \in \mathcal{X}_{A}$.

Let us check the uniqueness of the pair $(\mathfrak{a}, h)$. Let $I \in \mathcal{X}_{A}$ and assume that $(\mathfrak{a}, h)$ and ( $\mathfrak{a}^{\prime}, h^{\prime}$ ) satisfy the conditions (a), (b), (c), and (d) for the ideal $I$. Let $a \in R$. Then by condition (d), we have that $(a, 0) \in I$ if and only if $a \equiv 0 \bmod \mathfrak{a}$, whence $\mathfrak{a}=f^{-1}(I)=\mathfrak{a}^{\prime}$. Let $x \in L$ 
and choose $a \in R$ so that $h(x)=\bar{a}$. Then since $(a, x) \in I$, again by condition (d) we get $h(x)=\bar{a}=h^{\prime}(x)$. Hence $h=h^{\prime}$.

Let $I \in \mathcal{X}_{A}$ and take the pair $(\mathfrak{a}, h)$ for $I$ as is in Theorem 1.1. Let $\ell_{1}, \ell_{2}, \cdots, \ell_{n}$ be a system of generators for the $R$-module $L=(0):_{E}$ a and choose the elements $\left\{f_{i}\right\}_{1 \leq i \leq n}$ in $R$ so that $\bar{f}_{i}=h\left(\ell_{i}\right)$ for all $1 \leq i \leq n$. Then $I=\sum_{i=1}^{n} R \cdot\left(f_{i}, \ell_{i}\right)+\mathfrak{a} \times(0)$. We furthermore have the following.

COROllary 3.6. Let $I \in \mathcal{X}_{A}$ and choose the pair $(\mathfrak{a}, h)$ for $I$ as is in Theorem 1.1. Then the following conditions are equivalent.

(1) There exists $\theta \in \mathcal{H}$ satisfying the condition $h(x)=\overline{\theta(x)}$ for all $x \in L$.

(2) $I=\alpha\left(\mathfrak{a}^{\prime} \times\left[(0):_{E} \mathfrak{a}^{\prime}\right]\right)$ for some $\alpha \in \mathrm{Aut}_{R \text {-alg }} A$ and some ideal $\mathfrak{a}^{\prime}$ in $R$ with $\mathfrak{a}^{\prime 2}=(0)$.

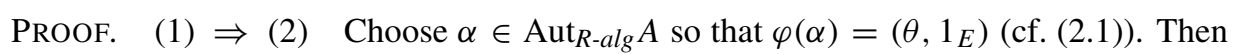
$\alpha(\mathfrak{a} \times L)=\{(a+\theta(x), x) \mid a \in \mathfrak{a}, x \in L\}$. Hence we get $I=\alpha(\mathfrak{a} \times L)$ by (1.1), because $\overline{\theta(x)}=h(x)$ for all $x \in L$.

$(2) \Rightarrow(1) \quad$ Because $\mathfrak{a}^{\prime}=f^{-1}(I)$, we have $\mathfrak{a}^{\prime}=\mathfrak{a}$ by (1.1) whence (0): $:_{E} \mathfrak{a}^{\prime}=L$. Let $x \in L$ and choose $a \in R$ so that $\bar{a}=h(x)$. Let $\varphi(\alpha)=(\rho, \tau)$ with $\rho \in \mathcal{H}$ and $\tau \in \mathrm{GL}_{R} E$ (cf. (2.1)). Then since $(a, x) \in I$ by (1.1), we have that $(a, x)=(b+\rho(y), \tau(y))$ for some $b \in \mathfrak{a}$ and $y \in L$. Hence $\bar{a}=\overline{\rho(y)}$ and $y=\tau^{-1}(x)$, so that $h(x)=\bar{a}=\overline{\theta(x)}$ where $\theta=\rho \tau^{-1} \in \mathcal{H}$.

\section{Example.}

Thanks to Theorem 1.1, in certain cases we are able to describe all the good ideals in $A=R \ltimes E$. Here let us explore one example.

Let $k[X, Y]$ be the polynomial ring in two variables over a field $k$ and let $R=k[X, Y] /\left(X^{3}-Y^{3}, X Y\right)$. We denote by $x, y$ the reduction of $X, Y \bmod \left(X^{3}-Y^{3}, X Y\right)$ and by $\mathfrak{n}$ the maximal ideal in $R$. Let $A=R \ltimes R$. Then the ideals $\mathfrak{a}$ in $R$ with $\mathfrak{a}^{2}=(0)$ are $(0),\left(x^{2}\right),\left(x^{3}\right),\left(\lambda x^{2}+y^{2}\right)(\lambda \in k)$, and $\left(x^{2}, y^{2}\right)$.

EXAMPLE 4.1. Assume that $\operatorname{ch} k \neq 2$. Then the set $\mathcal{X}_{A}$ consists of the following ideals:

\begin{tabular}{c|c}
\hline $\mathfrak{a}$ & $I \in \mathcal{X}_{A}$ with $\mathfrak{a}=f^{-1}(I)$ \\
\hline$(0)$ & $(0) \times R$ \\
\hline$\left(x^{2}\right)$ & $\left(x^{2}\right) \times\left(x^{2}, y\right)$ \\
\hline$\left(x^{3}\right)$ & $R \cdot\left(\lambda y^{2}, x\right)+R \cdot\left(-\lambda x^{2}, y\right)+R \cdot\left(x^{3}, 0\right)$ \\
& $(\lambda \in k)$ \\
\hline$\left(\lambda x^{2}+y^{2}\right)$ & $\left(\lambda x^{2}+y^{2}\right) \times(x-\lambda y) \quad(\lambda \neq 0)$ \\
$(\lambda \in k)$ & $\left(y^{2}\right) \times\left(x, y^{2}\right) \quad(\lambda=0)$ \\
\hline$\left(x^{2}, y^{2}\right)$ & $\left(x^{2}, y^{2}\right) \times\left(x^{2}, y^{2}\right)$ \\
\hline &
\end{tabular}


Hence $\sharp \mathcal{X}_{A}=2 \cdot \sharp k+3$ and so the set $\mathcal{X}_{A}$ is infinite, if so is $k$. If $\lambda \neq 0$, the ideal $I_{\lambda}=R \cdot\left(\lambda y^{2}, x\right)+R \cdot\left(-\lambda x^{2}, y\right)+R \cdot\left(x^{3}, 0\right)$ cannot be of the form $\alpha(\mathfrak{a} \times L)$ for any $\alpha \in$ Aut $_{R \text {-alg }} A$ and any ideal $\mathfrak{a}$ in $R$ with $\mathfrak{a}^{2}=(0)$, where $L=(0):_{E} \mathfrak{a}$. In fact, the pair $\left(\mathfrak{a}_{\lambda}, h_{\lambda}\right)$ corresponding to the ideal $I_{\lambda}$ is as follows: $\mathfrak{a}_{\lambda}=\left(x^{3}\right), L_{\lambda}=(0):_{R} \mathfrak{a}_{\lambda}=\mathfrak{n}$, and $h_{\lambda}(x)=\overline{\lambda y^{2}}, h_{\lambda}(y)=\overline{-\lambda x^{2}}$ in $R / \mathfrak{a}_{\lambda}$. Therefore if $\lambda \neq 0$, then by (3.6) $I_{\lambda} \neq \alpha(\mathfrak{a} \times L)$ for any $\alpha \in$ Aut $_{R \text {-alg }} A$ and for any ideal $\mathfrak{a}$ in $R$ with $\mathfrak{a}^{2}=(0)$, because $\mathcal{H}=(0)$ for this example.

Proof. The ring $R$ contains $\left\{1, x, y, x^{2}, y^{2}, x^{3}=y^{3}\right\}$ as $k$-basis. Let $\mathcal{F}$ denote the set of ideals $\mathfrak{a}$ in $R$ such that $\mathfrak{a}^{2}=(0)$. Then $\mathcal{F}=\left\{(0),\left(x^{2}\right),\left(x^{3}\right),\left(\lambda x^{2}+y^{2}\right)(\lambda \in k),\left(x^{2}, y^{2}\right)\right\}$, since $\mathfrak{a}^{2}=(0)$ if and only if $\mathfrak{a} \subseteq\left(x^{2}, y^{2}\right)$. Let $L=(0): \mathfrak{a}$ for $\mathfrak{a} \in \mathcal{F}$. Then

$$
L= \begin{cases}R & \text { if } \mathfrak{a}=(0) \\ \left(x^{2}, y\right) & \text { if } \mathfrak{a}=\left(x^{2}\right) \\ \mathfrak{n} & \text { if } \mathfrak{a}=\left(x^{3}\right) \\ (x-\lambda y) & \text { if } \mathfrak{a}=\left(\lambda x^{2}+y^{2}\right) \quad \text { and } \lambda \neq 0 \\ \left(x, y^{2}\right) & \text { if } \mathfrak{a}=\left(y^{2}\right) \\ \left(x^{2}, y^{2}\right) & \text { if } \mathfrak{a}=\left(x^{2}, y^{2}\right)\end{cases}
$$

For each $\mathfrak{a} \in \mathcal{F}$ we denote by $V$ the set of $R / \mathfrak{a}$-linear maps $h: L \rightarrow R / \mathfrak{a}$ satisfying the conditions $h(u) h(v)=0$ and $h(u) v+h(v) u=0$ for all $u, v \in L$. Let $W=\{h \in V \mid h$ satisfies conditions $(2)(b)$ and $(2)(c)$ in Theorem 1.1\}. We want to find all the elements in $W$. Here let us focus our attention on the case where $\mathfrak{a}=\left(x^{3}\right)$. The other cases are similarly proved and left to the reader.

We now look at the ideal $\mathfrak{a}=\left(x^{3}\right)$. Then $L=\mathfrak{n}$ and it has the presentation

$$
R^{3} \frac{\varphi}{\left[\begin{array}{ccc}
x^{2} & 0 & y \\
-y^{2} & x & 0
\end{array}\right]} R^{2} \stackrel{\pi}{\longrightarrow} L \longrightarrow 0
$$

where $\pi$ denotes the homomorphism defined by $\pi\left(\left[\begin{array}{c}a \\ b\end{array}\right]\right)=a x+b y$. Hence every homomorphism $h: L \rightarrow R / \mathfrak{a}$ is represented by a homomorphism $\psi=[\alpha \beta]: R^{2} \rightarrow R$ such that $\operatorname{Im}(\psi \varphi) \subseteq \mathfrak{a}$. As $\left[\begin{array}{ll}\alpha & \beta\end{array}\right]\left[\begin{array}{ccc}x^{2} & 0 & y \\ -y^{2} & x & 0\end{array}\right]=\left[\begin{array}{llll}a x^{2}-\beta y^{2} & \beta x & \alpha y\end{array}\right]$, the condition that $\operatorname{Im}(\psi \varphi) \subseteq \mathfrak{a}$ is equivalent to saying that $\alpha \in\left(x, y^{2}\right)$ and $\beta \in\left(x^{2}, y\right)$. Since $h(x)=\bar{\alpha}$ and $h(y)=\bar{\beta}$ in $R / \mathfrak{a}$, the homomorphism $h: L \rightarrow R / \mathfrak{a}$ is an element of $V$ if and only if $\alpha^{2}, \alpha \beta, \beta^{2} \in \mathfrak{a}$ and $\alpha x=\beta y=\alpha y+\beta x=0$ in $R$ (here we use the assumption that $\operatorname{ch} k \neq 2$ ). Let us write $\alpha=a x+b x^{2}+c y^{2}+d x^{3}$ and $\beta=a_{1} y+b_{1} x^{2}+c_{1} y^{2}+d_{1} x^{3}$ with $a, b, c, d, a_{1}, b_{1}, c_{1}$, and $d_{1} \in k$. Then $\alpha^{2}, \beta^{2} \in \mathfrak{a}$ if and only if $a=a_{1}=0$. When this is the case, we have $\alpha \beta \in \mathfrak{a}$, $\alpha x=b x^{3}, \beta y=c_{1} y^{3}$, and $\alpha y+\beta x=c y^{3}+b_{1} x^{3}$. Hence $h \in V$ if and only if $\alpha \equiv \lambda y^{2}$ and $\beta \equiv-\lambda x^{2} \bmod \mathfrak{a}$ for some $\lambda \in k$. Let us check that $V=W$, that is every $h \in V$ satisfies conditions (2)(b) and (2)(c) stated in Theorem 1.1. Since $\mathfrak{a}^{2}=(0)$, we may assume that $\lambda \neq 0$. Hence $h(L)=\left(x^{2}, y^{2}\right) / \mathfrak{a}$. We have $\left(x^{2}, y^{2}\right) \subseteq \operatorname{Ker}(h)$, because $h\left(x^{2}\right)=\bar{x} \cdot \overline{\lambda y^{2}}=0$ 
and $h\left(y^{2}\right)=\bar{y} \cdot \overline{-\lambda x^{2}}=0$ in $R / \mathfrak{a}$. Therefore $a b=0$ in $R$ for all $a, b \in R$ with $\bar{a}, \bar{b} \in h(L)$, since $\left(x^{2}, y^{2}\right)^{2}=(0)$. Let $a \in R$ with $\bar{a} \in h(L)$. Then $a \in\left(x^{2}, y^{2}\right)$ so that for all $b \in R$ we see $a b \in L=\mathfrak{n}$ and $h(a b)=0$, since $\left(x^{2}, y^{2}\right) \subseteq \operatorname{Ker}(h)$. Thus by Theorem 1.1 the good ideals $I$ in $A$ with $f^{-1}(I)=\left(x^{3}\right)$ are $I_{\lambda}=R \cdot\left(\lambda y^{2}, x\right)+R \cdot\left(-\lambda x^{2}, y\right)+R \cdot\left(x^{3}, 0\right)(\lambda \in k)$. We have that $I_{\lambda}=R \cdot\left(\lambda y^{2}, x\right)+R \cdot\left(-\lambda x^{2}, y\right)$ if $\lambda \neq 0$.

EXAMPLE 4.2. Assume that $\operatorname{ch} k=2$. Then the set $\mathcal{X}_{A}$ consists of the following ideals:

\begin{tabular}{c|c}
\hline $\mathfrak{a}$ & $I \in \mathcal{X}_{A}$ with $\mathfrak{a}=f^{-1}(I)$ \\
\hline$(0)$ & $R \cdot\left(a x^{2}+b y^{2}+c x^{3}, 1\right) \quad(a, b, c \in k)$ \\
\hline$\left(x^{2}\right)$ & $R \cdot\left(0, x^{2}\right)+R \cdot\left(a y^{2}, y\right)+R \cdot\left(x^{2}, 0\right) \quad(a \in k)$ \\
\hline$\left(x^{3}\right)$ & $R \cdot\left(a x^{2}+b y^{2}, x\right)+R \cdot\left(b x^{2}+c y^{2}, y\right)+R \cdot\left(x^{3}, 0\right) \quad(a, b, c \in k)$ \\
\hline$\left(\lambda x^{2}+y^{2}\right)$ & $R \cdot\left(a x^{2}+b y^{2}, x+\lambda y\right)+R \cdot\left(\lambda x^{2}+y^{2}, 0\right) \quad(a, b \in k)$ if $\lambda \neq 0$ \\
$(\lambda \in k)$ & $R \cdot\left(a x^{2}, x\right)+R \cdot\left(0, y^{2}\right)+R \cdot\left(y^{2}, 0\right) \quad(a \in k)$ if $\lambda=0$ \\
\hline$\left(x^{2}, y^{2}\right)$ & $\left(x^{2}, y^{2}\right) \times\left(x^{2}, y^{2}\right)$ \\
\hline
\end{tabular}

Let $I \in \mathcal{X}_{A}$ and let $(\mathfrak{a}, h)$ be the pair corresponding to $I$. Then, because $\mathcal{H} \cong\left(x^{2}, y^{2}\right)$ and every nonzero ideal in $R$ contains $\left(x^{3}\right)$, we have that $I \neq \alpha\left(\mathfrak{a} \times\left[(0):_{E} \mathfrak{a}\right]\right)$ for any $\alpha \in$ Aut $_{R \text {-alg }} A$ unless $\mathfrak{a}=(0)$ or $h=0$.

\section{References}

[BH] W. Bruns and J. Herzog, Cohen-Macaulay Rings, Cambridge Studies in Advanced Mathematics 39 (1993), Cambridge University Press.

[GIK] S. Goto, S. IAI, and M. KIM, Good ideals in Gorenstein local rings obtained by idealization, Proc. Amer. Math. Soc. 130 (2002), 337-344.

[GIW] S. Goto, S. IAI, and K. Watanabe, Good ideals in Gorenstein local rings, Trans. Amer. Math. Soc. 353 (2001), 2309-2346.

[GW] S. Goto and K. Watanabe, On graded rings, I, J. Math. Soc. Japan 30 (1978), 179-213.

[R] I. Reiten, The converse to a theorem of Sharp on Gorenstein modules, Proc. Amer. Math. Soc. 32 (1972), $417-420$.

Present Address:

Department of Mathematics, School of Science and Technology,

MEIJI UNIVERSITY, 214-8571 JAPAN. 